\title{
Correction to: Valencies of the lanthanides
}

\author{
David A. Johnson ${ }^{2} \cdot$ Peter G. Nelson ${ }^{1}$
}

Published online: 3 April 2018

(C) Springer Science+Business Media B.V., part of Springer Nature 2018

\section{Correction to: Found Chem (2018) 20:15-27 https://doi.org/10.1007/s10698-017-9291-6}

The authors regret that there are errors in equation (6) and subsequent discussion. The correct version is as follows:

$$
i=L\left[-\bar{E}_{\mathrm{f}}+\left(\Delta_{\mathrm{i}} p\right) \bar{E}_{\mathrm{p}}-\left(\Delta_{\mathrm{i}} q\right) \bar{E}_{\mathrm{q}}+\left(\Delta_{i} c_{3}\right) \bar{E}^{3}\right]+(5 / 2) R T
$$

The values of $\Delta_{\mathrm{i}} p,-\Delta_{\mathrm{i}} q$, and $\Delta_{\mathrm{i}} c_{3}$ are given in Table 4 . The second of these determines the loss of exchange energy and peaks at $\mathrm{f}^{7}$.

As with the tetrad effect, the irregularities in $\Delta_{\mathrm{i}} c_{3}$ centred on the quarter- and the threequarter full shell are associated with the ability of electrons to keep apart better if their $m_{l}$ values have the same sign rather than opposite. This eases the general increase in interelectronic repulsion in the first quarter of the series, where electrons have $m_{l}$ values of the same sign, relative to the second quarter, where they have both positive and negative values (Table 2). On ionization, this easement is lost in the first quarter and gained in the second (Table 4); similarly in the third quarter over the fourth quarter.

Errors have subsequently been identified in the original publication. Table 5 was incorrectly published and the corrected table is given below:

The original article can be found online at https://doi.org/10.1007/s10698-017-9291-6.

Peter G. Nelson

p.g.nelson@hull.ac.uk

1 Department of Chemistry, University of Hull, Hull HU6 7RX, UK

2 Department of Chemistry, The Open University, Milton Keynes MK7 6AA, UK 
Table 4 Values of the coefficients in Eq. (6)

\begin{tabular}{lrlr}
\hline Process & \multicolumn{1}{c}{$\Delta_{\mathrm{i}} p$} & $-\Delta_{\mathrm{i}} q$ & $\Delta_{\mathrm{i}} c_{3}$ \\
\hline $\mathrm{f}^{1} \rightarrow \mathrm{f}^{0}$ & 0 & 0 & 0 \\
$\mathrm{f}^{2} \rightarrow \mathrm{f}^{1}$ & -1 & 1 & +9 \\
$\mathrm{f}^{3} \rightarrow \mathrm{f}^{2}$ & -2 & 2 & +12 \\
$\mathrm{f}^{4} \rightarrow \mathrm{f}^{3}$ & -3 & 3 & 0 \\
$\mathrm{f}^{5} \rightarrow \mathrm{f}^{4}$ & -4 & 4 & -12 \\
$\mathrm{f}^{6} \rightarrow \mathrm{f}^{5}$ & -5 & 5 & -9 \\
$\mathrm{f}^{7} \rightarrow \mathrm{f}^{6}$ & -6 & 6 & 0 \\
$\mathrm{f}^{8} \rightarrow \mathrm{f}^{7}$ & -7 & 0 & 0 \\
$\mathrm{f}^{9} \rightarrow \mathrm{f}^{8}$ & -8 & 1 & +9 \\
$\mathrm{f}^{10} \rightarrow \mathrm{f}^{9}$ & -9 & 2 & 0 \\
$\mathrm{f}^{11} \rightarrow \mathrm{f}^{10}$ & -10 & 3 & -12 \\
$\mathrm{f}^{12} \rightarrow \mathrm{f}^{11}$ & -11 & 4 & -9 \\
$\mathrm{f}^{13} \rightarrow \mathrm{f}^{12}$ & -12 & 5 & 0 \\
$\mathrm{f}^{14} \rightarrow \mathrm{f}^{13}$ & -13 & 6 &
\end{tabular}

Table 5 Nature of apparently bivalent compounds

\begin{tabular}{|c|c|c|c|c|c|c|c|c|c|c|c|c|c|c|}
\hline & La & Ce & Pr & Nd & Pm & Sm & Eu & Gd & Tb & Dy & Ho & Er & Tm & Yb \\
\hline LnI $_{2}$ & $\mathrm{M}$ & $\mathrm{M}$ & $\mathrm{M}$ & $\mathrm{S}$ & $\mathrm{S}$ & $\mathrm{S}$ & $\mathrm{S}$ & $\mathrm{M}$ & $-^{\mathrm{b}}$ & $\mathrm{S}$ & $-^{\mathrm{b}}$ & $-^{\mathrm{b}}$ & $\mathrm{S}$ & $\mathrm{S}$ \\
\hline LnTe & $\mathrm{M}$ & $\mathrm{M}$ & $\mathrm{M}$ & $\mathrm{M}$ & $\mathrm{M}$ & $\mathrm{S}$ & $\mathrm{S}$ & $\mathrm{M}$ & $\mathrm{M}$ & $\mathrm{M}$ & $\mathrm{M}$ & $\mathrm{M}$ & $\mathrm{S}$ & $\mathrm{S}$ \\
\hline LnSe & $\mathrm{M}$ & $\mathrm{M}$ & $\mathrm{M}$ & $\mathrm{M}$ & $\mathrm{M}$ & $\mathrm{S}$ & $\mathrm{S}$ & $\mathrm{M}$ & $\mathrm{M}$ & $\mathrm{M}$ & $\mathrm{M}$ & $\mathrm{M}$ & $\mathrm{X}$ & $\mathrm{S}$ \\
\hline $\mathrm{LnS}^{\mathrm{N}}$ & $\mathrm{M}$ & $\mathrm{M}$ & $\mathrm{M}$ & $\mathrm{M}$ & $\mathrm{M}$ & $\mathrm{S}$ & $\mathrm{S}$ & $\mathrm{M}$ & $\mathrm{M}$ & $\mathrm{M}$ & $\mathrm{M}$ & $\mathrm{M}$ & $\mathrm{M}$ & $\mathrm{S}$ \\
\hline $\mathrm{LnH}_{2}$ & $\mathrm{M}$ & $\mathrm{M}$ & $\mathrm{M}$ & $\mathrm{M}$ & $\mathrm{M}$ & $\mathrm{M}$ & $\mathrm{S}$ & $\mathrm{M}$ & $\mathrm{M}$ & $\mathrm{M}$ & $\mathrm{M}$ & $\mathrm{M}$ & $\mathrm{M}$ & $\mathrm{S}$ \\
\hline $\mathrm{LnC}_{2}$ & $\mathrm{M}$ & $\mathrm{M}$ & $\mathrm{M}$ & $\mathrm{M}$ & $\mathrm{M}$ & $\mathrm{M}$ & $\mathrm{S}$ & $\mathrm{M}$ & $\mathrm{M}$ & $\mathrm{M}$ & $\mathrm{M}$ & $\mathrm{M}$ & $\mathrm{M}$ & $\mathrm{X}$ \\
\hline
\end{tabular}

At room temperature and pressure

$S$ salt-like, $M$ metallic, $X$ mixed

${ }^{\text {a}}$ Predicted (from Fig. 6)

${ }^{b}$ Attempts to prepare $\mathrm{LnI}_{2}$ by the reaction of $\mathrm{Ln}$ with $\mathrm{LnI}_{3}$, which were successful at dysprosium, were unsuccessful in these cases (Johnson and Corbett 1970) 